\title{
Comparison of Tensile Bond Strength of Addition Silicone with Different Custom Tray Materials Using Different Retentive Methods
}

\author{
Rupali V Patil ${ }^{1}$, Vasantha Vijayraghavan ${ }^{2}$, Madhurakad Jadhav ${ }^{3}$, Shweta Jajoo ${ }^{4}$, Sneha Desai ${ }^{5}$, Chetana Jagtap $^{6}$
}

\begin{abstract}
Aim: To compare the bond strength of addition silicone with different commonly used custom tray materials by means of different retentive methods (mechanical, chemical, and a combination of chemical and mechanical methods).

Materials and methods: Fabrications of 90 samples of different tray resin materials were done using an aluminum mold. They were divided into three main groups. Perforations, adhesive application, and a combination of both were done according to the grouping of samples. Polyvinyl siloxane material (medium body) was loaded over the samples. A universal testing machine with a crosshead speed of $5 \mathrm{~mm} / \mathrm{minute}$ was used to determine the tensile bond strength of tray resin samples to medium body impression material. Based on these values, Student's-test, group statistics, and ANOVA test were used for statistical analysis.

Results: Visible light cure (VLC) resin showed the highest bond strength in chemicomechanical methods. This was followed by repair resin material. Tray resin material showed poor bond strength in all three retentive methods. The mechanical method was the least retentive in all three resin materials.

Clinical significance: VLC tray resin material can be used with chemical and mechanical retention in clinical situations to make predictably accurate elastomeric impressions.

Conclusion: It was concluded that VLC tray resin shows good bond strength with polyvinyl siloxane impression material when both mechanical perforations and adhesive applications were done.

Keywords: Acrylic resin, Addition silicone, Tensile bond strength, Tray adhesive, Tray repair material, Visible light cure resin.

The Journal of Contemporary Dental Practice (2021): 10.5005/jp-journals-10024-3009
\end{abstract}

\section{INTRODUCTION}

Polyvinyl siloxane impression materials are among the most popular elastomeric impression materials used in dentistry. Due to their excellent dimensional stability, hydrophilic nature, accuracy, excellent elastic recovery, coupled with good tear strength, they are the most commonly used material in fixed prosthodontics and implant dentistry. An important, often less discussed, factor in elastomeric impression material is the adhesion of an elastomer to the impression tray when the impression is removed from the undercuts and the oral structures. ${ }^{1}$

The use of the custom tray to enhance the dimensional accuracy of the impression and, subsequently, the fabricated prosthesis has been validated by numerous studies by allowing a more uniform thickness of the impression material. ${ }^{2}$ Different custom tray materials are available such as the autopolymerizing resin tray material, different visible light cure (VLC) tray material, and heatactivated acrylic resin. ${ }^{3,4}$

During the tray removal, combinations of stresses develop within the impression-materials' adhesivetray systems. If the tray is removed perpendicular to the occlusal plane, tensile and shear stress dominate; in the palatal area, the bond will be stressed by the tensile force. If the material pulls away from the tray during its removal from the mouth, the complete material will fail to return to its original shape resulting in faulty casting. ${ }^{5}$

Hence, the bond strength of the impression material to a tray is essential. ${ }^{6}$ Several investigators have concluded that the most consistently accurate impressions were obtained with the

\footnotetext{
1,2 Department of Prosthodontics Crown and Bridge, Bharati Vidyapeeth Dental College and Hospital, Pune, Maharashtra, India

${ }^{3}$ Department of Orthodontics, Bharati Vidyapeeth Dental College and Hospital, Pune, Maharashtra, India

${ }^{4-6}$ Department of Pedodontics and Preventive Dentistry, Bharati Vidyapeeth Dental College and Hospital, Pune, Maharashtra, India

Corresponding Author: Rupali V Patil, Department of Prosthodontics Crown and Bridge, Bharati Vidyapeeth Dental College and Hospital, Pune, Maharashtra, India, Phone: +91 9850077001, e-mail: dr.rupalipatil7001@gmail.com
}

How to cite this article: Patil RV, Vijayraghavan V, Jadhav $M$, et al. Comparison of Tensile Bond Strength of Addition Silicone with Different Custom Tray Materials Using Different Retentive Methods. J Contemp Dent Pract 2021;22(3):278-283.

Source of support: Nil

Conflict of interest: None

adhesive-line perforated custom tray, thus, combining mechanical retention with chemical retention.

However, todate, no study has been done for the comparative evaluation of these two methods or the combination of these two methods with different custom tray materials.

The reasonably good bond strength between the tray and the impression material is essential to produce an accurate cast for successful prosthetic treatment. Hence, this study has been done to compare the bond strength of addition silicone to different 
commonly used custom tray materials by means of different retentive methods (mechanical, chemical, and chemicomechanical). The most retentive surface preparation (i.e., adhesive application, making perforations, or a combination of both) on three different custom tray materials was also determined. A universal testing machine evaluated the tensile bond strength between the impression material and the different resin trays.

\section{Materials and Methods}

This was an in vitro experimental study. The total number of samples in the study was 90 . They were divided into three groups of 30 samples each for tray material resin, tray repair resin, and VLC resin. The grouping of the samples was done, and it is shown in the Fig. 1.

\section{Fabrication of the Sample Mold}

Three aluminum dies were fabricated to prepare the main tray, cover tray, and perforations in the tray. The main die was designed in such a way that it will make a tray having $30 / 30 \mathrm{~mm}$ testing surface area with $2 \mathrm{~mm}$ depth and 10/10 $\mathrm{mm}$ borders. A cover tray die was designed in such a way that a $40 / 40 \mathrm{~mm}$ tray will be made (Fig. 2). A perforated die was prepared in such a way with holes of $2 \mathrm{~mm}$ diameter and $5 \mathrm{~mm}$ in depth that were $5 \mathrm{~mm}$ away from each other.

\section{Fabrication of Samples}

All the resin blocks of acrylic resin (MP Sai Enterprises, Mumbai) and acrylic repair resin (DPI RR Cold Cure) were prepared in an aluminum mold. The resin material was manipulated according to the manufacturer's instructions and packed into the mold. Lids were slid over the mold so that excess material came out from the center. Then, the threaded portion of the screw was inserted into the resin at the center. Once the material had been set, the lids were removed, and the screws were tightened. Then the samples were removed. The same procedure was done for the cover trays except for the screw insertion. The samples were stored at room temperature for 24 hours. $^{7-9}$
VLC material (Plaque Photo, WP Dental) is available in a sheet form. A soft sheet was adapted into a mold. Lids were slid over the mold so that excess material comes out from the center, and the threaded portion of the screw is inserted into the material. VLC resin trays were cured in a light chamber with the blue light of 400 to $500 \mathrm{~nm}$ from a high-intensity quartz halogen bulb for 9 minutes.

After 9 minutes, the mold was removed from the curing unit, and the lids were separated. The sample was removed by tightening the screws. It was inverted and cured for 9 minutes. Due to the thickness of the material, it does not cure completely. In the same manner, the cover trays were made, except for the screw insertion.

The samples that were to be tested for mechanical retention (30 in number) and chemicomechanical retention (30 in number) were perforated by using the perforated die and straight fissure bur number 8 . It was repeated for the cover trays.

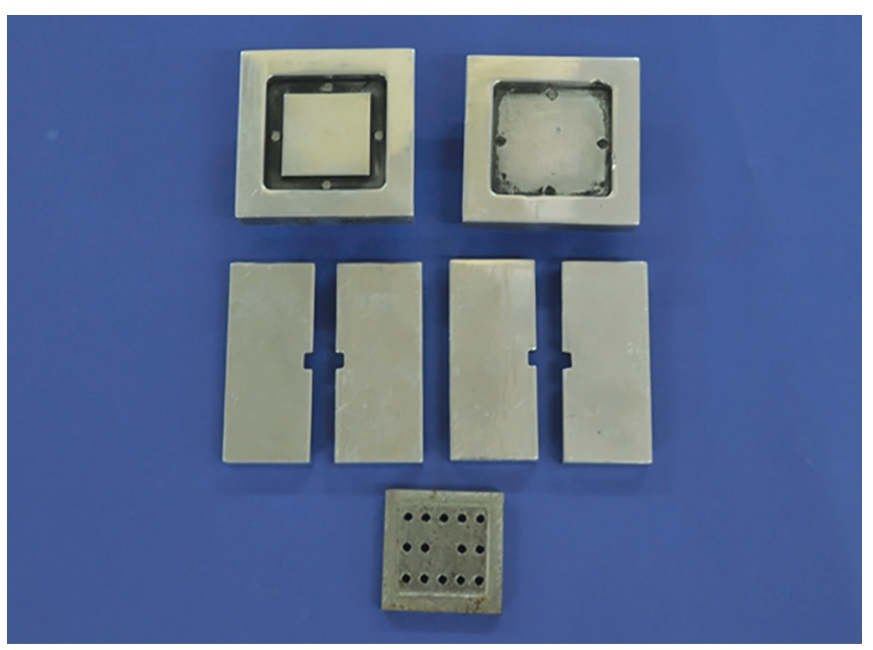

Fig. 2: Aluminum die

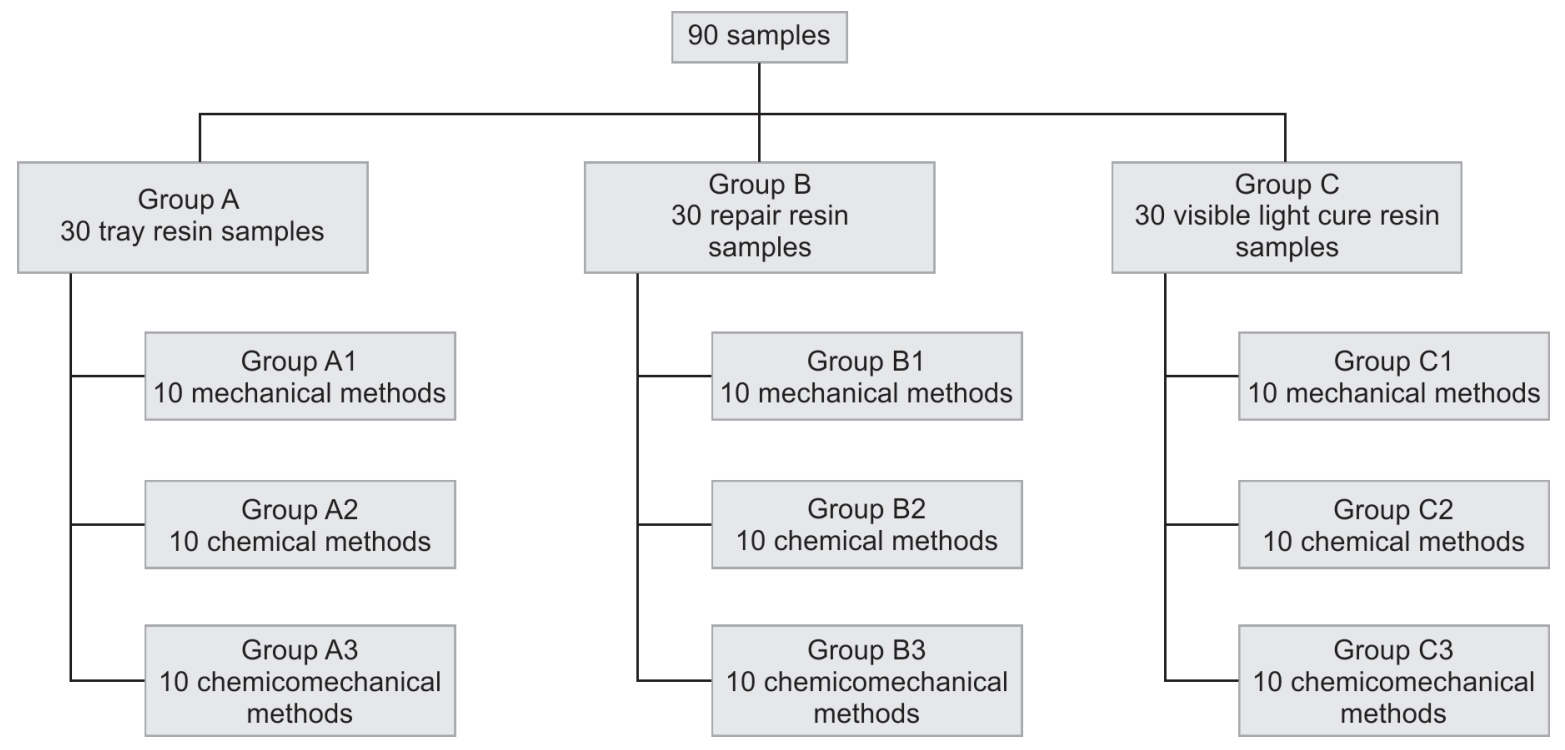

Fig. 1: Grouping of samples 
The samples for chemical retention were handled by a single thin layer of tray adhesive application with a brush over the surface of acrylic resin trays. They were allowed to dry at room temperature for 15 minutes according to the manufacturer's instructions. ${ }^{10}$ The adhesive was confined to the tested surface (Fig. 3). It was repeated for the cover trays. For the combination method, the samples' perforations were done followed by adhesive applications.

The impression material (Monophase Aquasil) was mixed according to the manufacturer's instructions. It was loaded onto the resin trays. Both the resin trays were approximated till the rims of the tray touched each other completely, and it was ensured that a uniform $2 \mathrm{~mm}$ thickness of the material was present between the block and fixture. Excess material that had flown out was cut with a scalpel (Fig. 4).

When the material had set completely, the sample was attached to the universal testing machine (Fig. 5). Blinding was done to obtain unbiased results. Each sample was tested under a crosshead speed of $5 \mathrm{~mm} /$ minute. The maximum load at which the material gets debonded was recorded. The tensile strength was calculated by dividing the maximum load by the cross-sectional area. The tensile bond strength was recorded in MPa.

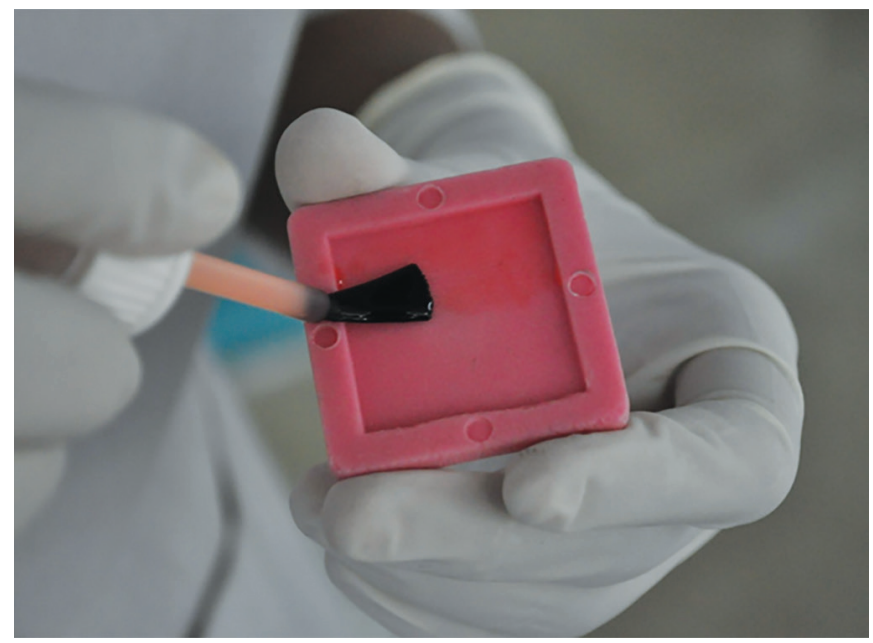

Fig. 3: Application of the adhesive

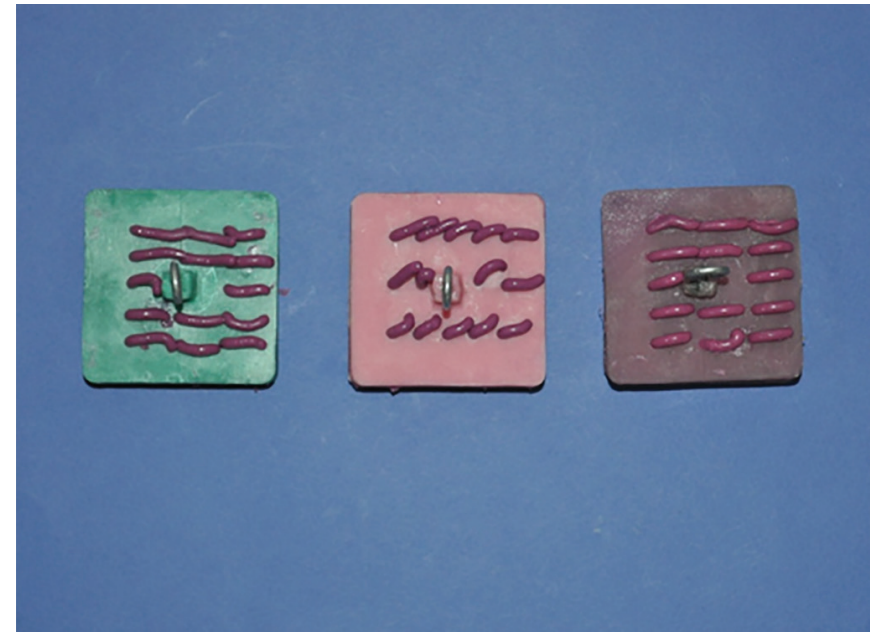

Fig. 4: Material loaded in the samples

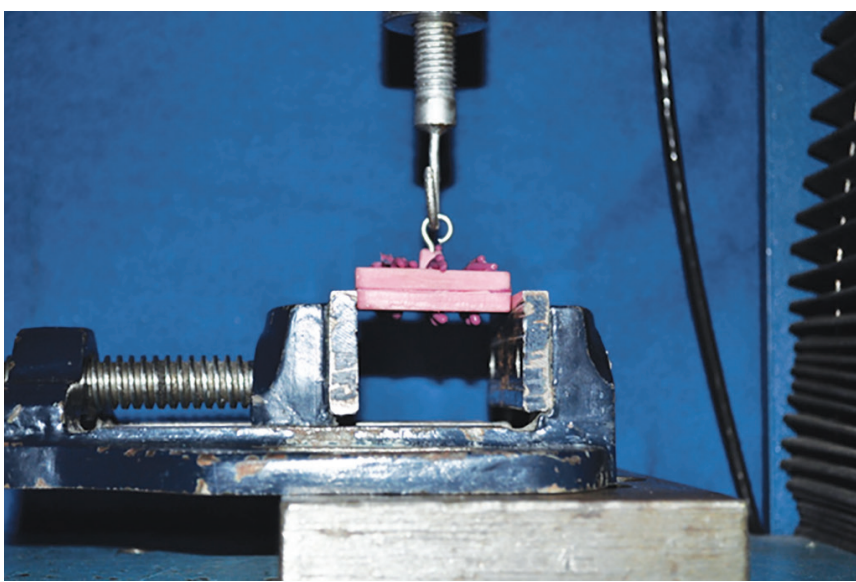

Fig. 5: Samples tested in the universal testing machine

\section{Statistical Analysis}

The readings obtained were statistically analyzed, using Student $t$-test, group statistics, and ANOVA tests. The mean and standard deviation of the three groups are mentioned in Tables 1 to 3 . Results of the intergroup comparison are depicted in Graphs 1 to 3.

\section{Discussion}

With the advent of polymer technology, the dental profession witnessed a group of synthetic rubbery materials called elastomers. ${ }^{7,8}$ Among elastomers, due to its excellent physical properties and versatility, the polyvinyl siloxane impression material is advocated for making impressions for removable and fixed prosthodontics. Brown discussed the factors affecting the accuracy of an impression and stated that the selection of material and the technique used in making the impression are the two vital factors that are under the direct control of the operator. ${ }^{11}$

According to Phillips et al., and Eames et al., the dimensional changes of addition siloxane are minimized when the material is used in the thickness of 2 to $3 \mathrm{~mm}$. ${ }^{8,12}$ The proposed $2 \mathrm{~mm}$ thickness of the elastomeric impression material can be best achieved through the use of custom impression trays. Also, a custom tray is advisable for procedures requiring precision and accuracy rather than a stock tray to minimize the dimensional changes and resulting inaccuracies. ${ }^{13,14}$

The more popular ones among custom tray materials are autopolymerizing resin and light-activated resin because of their availability and ease of use. ${ }^{15}$ VLC tray resin demonstrates less porosity, and it has less leachable unreacted residual monomers. The fabrication and modification with the VLC resin can be performed more quickly than others. ${ }^{16}$

In view of the dislodging forces occurring during the removal of the tray from the mouth, it is extremely important that the adhesive bond formed between the impression material and the tray must be of sufficient strength to withstand the forces generated during the removal of the set material. ${ }^{5,17}$

Mechanical retention is obtained by the presence of undercuts and perforations in the tray into which the impression material becomes locked. However, no single technique, i.e., mechanical or chemical has been found to be satisfactory when used alone. The mechanical retention between the impression material and the tray is difficult to achieve at the periphery of the tray. Therefore, adhesives are useful adjuncts in these areas. To date, no study has 
Table 1: Comparison of bond strength with respect to tray material by the mechanical method, chemical method, and mechanical + chemical method

\begin{tabular}{lllll}
\hline & & & \multicolumn{2}{c}{$95 \%$ confidence interval for mean } \\
\cline { 3 - 5 } Method & Number of cases & Strength of bonding (mean \pm SD) & Lower bound & Upper bound \\
\hline Mechanical & 10 & $0.214 \pm 0.022$ & 0.198 & 0.230 \\
Chemical & 10 & $0.243 \pm 0.080$ & 0.185 & 0.300 \\
Mechanical + chemical & 10 & $0.297 \pm 0.035$ & 0.272 & 0.005 \\
\hline
\end{tabular}

Table 2: Comparison of bond strength with respect to repair material by the mechanical method, chemical method, and mechanical + chemical method

\begin{tabular}{|c|c|c|c|c|c|}
\hline \multirow[b]{2}{*}{ Method } & \multirow[b]{2}{*}{ Number of cases } & \multirow[b]{2}{*}{ Strength of bonding (mean $\pm S D)$} & \multicolumn{2}{|c|}{ 95\% confidence interval for mean } & \multirow[b]{2}{*}{ pvalue } \\
\hline & & & Lower bound & Upper bound & \\
\hline Mechanical & 10 & $0.226 \pm 0.068$ & 0.177 & 0.274 & $<0.001$ \\
\hline Chemical & 10 & $0.309 \pm 0.051$ & 0.272 & 0.346 & \\
\hline Mechanical + chemical & 10 & $0.326 \pm 0.067$ & 0.278 & 0.374 & \\
\hline
\end{tabular}

Table 3: Comparison of bond strength with respect to visible light cure material by the mechanical method, chemical method, and mechanical + chemical method

\begin{tabular}{lllll}
\hline & & & \multicolumn{2}{c}{$95 \%$ confidence interval for mean } \\
\cline { 3 - 5 } Method & Number of cases & Strength of bonding (mean \pm SD) & Lower bound & Upper bound \\
\hline Mechanical & 10 & $0.268 \pm 0.051$ & 0.232 & 0.304 \\
Chemical & 10 & $0.359 \pm 0.040$ & 0.330 & 0.388 \\
Mechanical + chemical & 10 & $0.409 \pm 0.043$ & 0.378 & 0.440 \\
\hline
\end{tabular}

been done to evaluate the combination of mechanical and chemical methods under ideal testing conditions.

The three most commonly used custom tray fabrication materials were used for the fabrication of the custom tray, viz., tray material (group A), repair material (group B), and VLC resin (group C).

The trays were fabricated using the die. For the perforation of the fabricated trays, a perforated metal plate die containing $2 \mathrm{~mm}$ holes at a distance of $2 \mathrm{~mm}$ from each other was used as a guide. This helped in the standardization of all the testing samples. In each group, perforations were done on 10 samples. This is in agreement with Fusayama. ${ }^{18}$ In the chemical retention method, a tray adhesive according to the manufacturer's instruction was used as suggested by Samman and Fletcher. ${ }^{19}$ So, a single thin coat of manufacturerrecommended tray adhesive for addition silicone was applied on the testing surface of the tray and along the vertical walls since the polymerization shrinkage is more at the peripheries than at the center of the tray. In the third method of retention, both perforations as well as adhesive application were done. This treatment was done on 10 trays for each group.

The impression material chosen for the study was monophase polyvinyl siloxane, also known as medium body addition silicone due to its various positive aspects. ${ }^{20-22}$

The samples in each group were tested using the universal testing machine.

The tensile bond strength was least with mechanical perforations in all groups. When the tensile bond strength of the mechanical method was compared between all the three groups, i.e., A1 (0.214 MPa), B1 (0.226 MPa), and C1 (0.268 MPa), no statistically significant difference was observed (Graph 1). Sammen recommended that perforations were effective primarily against the shear stresses. ${ }^{19}$ Shear stress is the ratio of force to the original cross-sectional area parallel to the direction of the force. It is a result when two forces are directly parallel to each other. When force is applied on the tray shear stresses, the tensile stress will act at the junction between the impression material and the tray. ${ }^{8}$ Resistance to the shear stress is provided by the material that is locked in the vertical walls of the perforation, and tensile force is resisted by the material that extruded through the perforations and coalescence on the outer surface.

When the three groups with the chemical method of retention were tested for tensile bond strength, i.e., group A2 (tray material), B2 (repair material), and C2 (VLC resin), a significant difference was observed (Graph 2). The tensile bond strength was maximum in the visible resin tray $(0.359 \mathrm{MPa})$, followed by repair resin $(0.309 \mathrm{MPa})$, and tray resin $\mathrm{A} 2(0.243 \mathrm{MPa})$. Tensile bond strength was least in the tray resin material, and it was most in VLC material.

The adhesive for silicone contains poly(dimethylsiloxane) or similar reactive silicone and tetraethyl orthosilicate to create a physical bond between the tray and the polydimethyl siloxane impression material. ${ }^{8}$ It is believed that on the application of the adhesive, the carrier solvents swell the outermost surface of the tray, allowing the adhesive to penetrate and interact with the tray material. The solvent then evaporates leaving the entire surface 


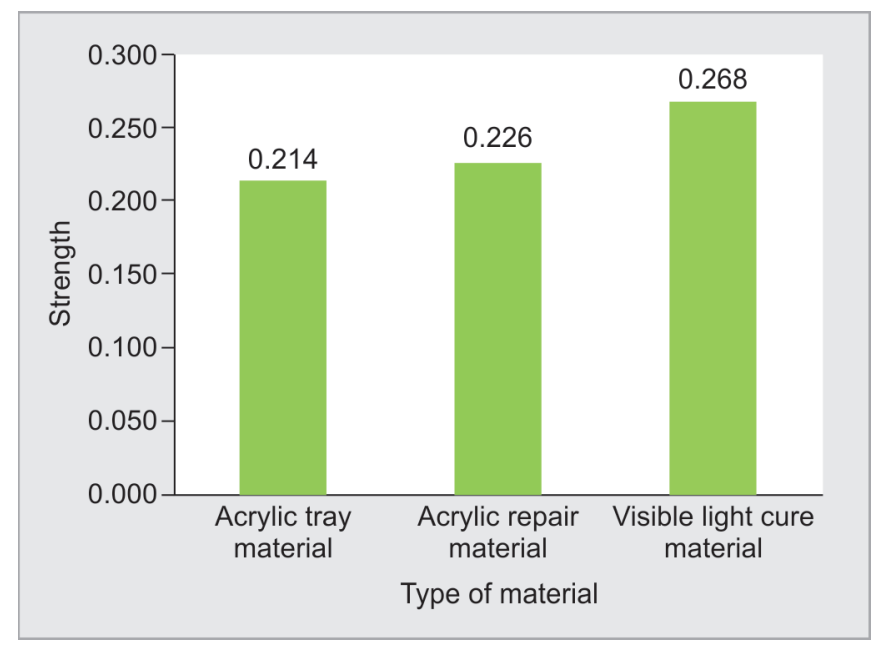

Graph 1: Comparison of bond strength between tray material, repair material, and visible light cure material by the mechanical method

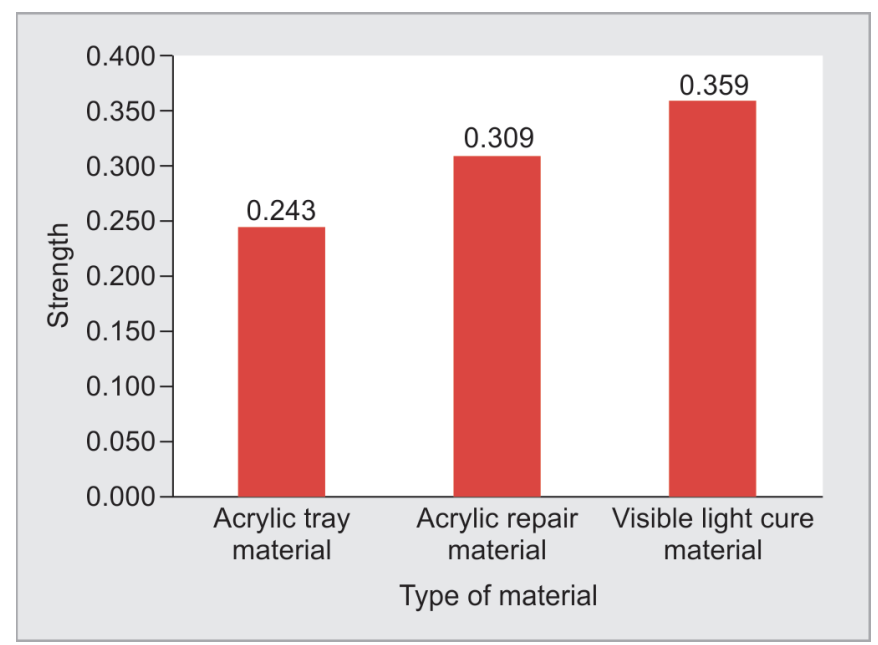

Graph 2: Comparison of bond strength between acrylic tray material, acrylic repair material, and visible light cure material by the chemical method

covered with the tray adhesive, which is retained on the molecular network of the superficial layer of the tray material. Impression retention is related to the chemistry of the adhesive agents and the surface chemistry of the resin tray material. ${ }^{23}$

The tetraethyl orthosilicate (TEOS) of the tray adhesive is a chemical compound with the formula $\mathrm{Si}\left(\mathrm{OC}_{2} \mathrm{H}_{5}\right)_{4}$. This molecule consists of four ethyl groups attached to the orthosilicate ion. According to Bulla and Morimoto, TEOS is mainly used as a crosslinking agent in silicone polymers and also a precursor of silica dioxide. ${ }^{6}$ Tetraethyl orthosilicate has a remarkable property of easily converting into silica dioxide. This reaction occurs upon the addition of water.

$$
\mathrm{Si}\left(\mathrm{OC}_{2} \mathrm{H}_{5}\right)_{4}+2 \mathrm{H}_{2} \mathrm{O}=\mathrm{SiO}_{2}+4 \mathrm{C}_{2} \mathrm{H}_{5} \mathrm{OH}
$$

The side product is ethanol. This reaction proceeds via a series of condensation reactions that convert the TEOS molecule into a mineral-like solid via the formation of Si-O-Si linkages. According to Skinner, the tray material consists of inorganic fillers, i.e., French

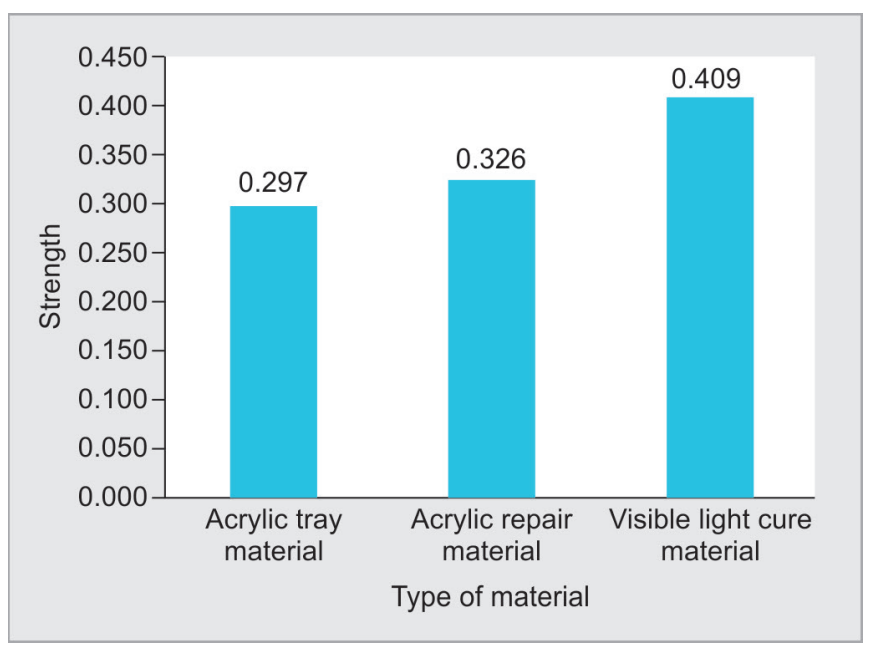

Graph 3: Comparison of bond strength between tray material, repair material, and visible light cure material by the mechanical + chemical method

chalk, a repair material, consists of silica fillers in the form of glass and beads, and VLC consists of microfilled silica. ${ }^{8}$ Therefore, the interaction of TEOS with silica of VLC is the maximum as the filler content is the highest. This is in accordance with results in which the maximum tensile strength with tray adhesives was found with VLC resin followed by repair material and the least in tray material. Chi et al. postulated that better adherence of polyvinyl siloxane to VLC tray material than to acrylic resin tray material may be the result of the difference in the solubility of the tray material in the solvent of the impression's adhesive. ${ }^{24}$

When the three groups of chemicomechanical methods, i.e., groups A3, B3, and C3, were compared for tensile bond strength, the maximum was seen in the case of VLC resin $(0.409 \mathrm{MPa})$ followed by repair resin $(0.326 \mathrm{MPa})$, and finally, tray resin $(0.297 \mathrm{MPa})$. The difference was highly statistically significant $(p<0.001)$ (Graph 3).

Samman and Fletcher suggested that the combination of the chemical and mechanical retention provides greater resistance and ultimately results in higher bond strength. ${ }^{19}$

The following are the limitations of the study: When an impression is removed from the mouth both the tensile and shear forces act on the interface of the impression and the tray. In this study, only the tensile bond strength has been tested. Shear load testing could have been incorporated. Furthermore, a scanning electron microscopic study is required to know whether the failure of the bond is adhesive or cohesive in nature.

\section{Conclusion and Clinical Significance}

Polyvinyl siloxane shows significantly higher bond strength to the VLC tray than tray material and repair tray material as supported by various studies. VLC tray material has many advantages over acrylic tray material.

The combination of both mechanical and chemical retention offers the most accurate impressions and the prostheses, thus indicating enhanced retention over the use of either system alone. Hence, within the limitations of this study, it can be concluded that VLC tray resin shows good bond strength with polyvinyl siloxane impression material when both mechanical perforations and adhesive applications were done. 


\section{References}

1. Wassell RW, Ibbetson RJ. Accuracy of polyvinyl siloxane impression made with standard and reinforced stock trays. J Prosthet Dent 1991;65(6):748-757. DOI: 10.1016/s0022-3913(05)80006-x.

2. Lacy AM, Fukui H. Bellman T, et al. Time dependent accuracy of elastomeric impression materials. Part II: Polyether, polysulphide and polyvinylsiloxane. J Prosthet Dent 1981;45(3):329-333. DOI: 10.1016/0022-3913(81)90400-5.

3. Dixon DL, Breeding LC, Mosely JP. Custom impression trays. Part II: Removal forces. J Prosthet Dent 1994;71(3):316-318. DOI: 10.1016/0022-3913(94)90474-X.

4. Wang RR, Nguyen T, Boyle AM. The effect of tray material and surface condition on the shear bond strength of impression materials. J Prosthet Dent 1995;74(5):563-568. DOI: 10.1016/s0022-3913(05)80344-0.

5. Bomberg TJ, Goldfogel MH, Hoffman W, et al. Consideration for adhesion of impression materials to impression trays. J Prosthet Dent 1988;60(6):681-684. DOI: 10.1016/0022-3913(88)90398-8.

6. Bulla DAP, Morimoto NI. Deposition of thick TEOS PECVD silicon oxide layers for integrated optical waveguide applications. J Thin Solid Films 1998;334(1-2):60-64. DOI: 10.1016/S0040-6090(98)01117-1.

7. Pagniano RP, Scheid RC, Clowson RL, et al. Linear dimensional change of acrylic resins used in fabrication of custom trays. J Prosthet Dent 1982;47(3):279-284. DOI: 10.1016/0022-3913(82)90157-3.

8. Phillips RW. Skinner science of dental materials. 8th ed. Philadelphia; 1991.

9. Fehling AW, Hesby RA, Pelleu GB. Dimensional stability of auto polymerizing acrylic resin impression trays. J Prosthet Dent 1986;55(5):592-597. DOI: 10.1016/0022-3913(86)90038-7.

10. Dixon DL, Breeding LC, Brown JS. The effect of custom tray material type and adhesive drying type on tensile bond strength of an impression material/adhesive system. Int J Prosthodont 1994;7(2):129-133. PMID: 8003192.

11. Brown D. Factors affecting the dimensional stability of elastomeric impression materials. J Dent 1973;1(6):265-274. DOI: 10.1016/03005712(73)90103-6.

12. Eames WB, Sieweke JC, Walance SW, et al. Elastomeric impression material: Effect of bulk on accuracy. J Prosthet Dent 1979;41(3):304307. DOI: 10.1016/0022-3913(79)90013-1.
13. Bomberg PJ, Hatch RA, Hoffman W. Impression material thickness in stock and custom trays. J Prosthet Dent 1985;54(2):170-172. DOI: 10.1016/0022-3913(85)90278-1.

14. Gordon GE, Johnson GH, Drennon DG. The effect of tray selection and accuracy of impression of tray materials. J Prosthet Dent 1990;63(1):12-15. DOI: 10.1016/0022-3913(90)90257-d.

15. Abdullah MA, Talic YF. The effect of custom tray material type and fabrication technique on tensile bond strength of impression material adhesive system. J Oral Rehabil 2003;30(3):312-317. DOI: 10.1046/j.1365-2842.2003.01023.x.

16. Devlin H, Cash AJ, Watts DC. Mechanical behavior and structure of light cured special tray materials. J Dent 1995;23(4):255-259. DOI: 10.1016/0300-5712(95)91191-O.

17. Tjan AH, Nemetz $H$, Nguyen LT, et al. Effect of tray space on the accuracy of monophasic polyvinylsiloxane impressions. J Prosthet Dent 1992;68(1):19-28.

18. Fusayama T, Nakazato M. The design of stock trays and the retention of irreversible hydrocolloid impressions. J Prosthet Dent 1969;21(2):136-142. DOI: 10.1016/0022-3913(69)90086-9.

19. Samman JM, Fletcher A. A study of impression tray adhesive. Quintessence Int 1985;16(4):305-309. PMID: 3892569.

20. Chee WW, Donovan TE. Polyvinyl siloxane impression material. A review properties and techniques. J Prosthet Dent 1992;68(5):728-732. DOI: 10.1016/0022-3913(92)90192-d.

21. Klooster J, Logan GI, Tjan AH. Effects of strain rate on the behavior of elastomeric impression. J Prosthet Dent 1991;66(3):292-298. DOI: 10.1016/0022-3913(91)90252-r.

22. Millar BJ, Dunne SM, Robinson PB. In vitro study of number of surface defects in monophase and two phase addition silicone. J Prosthet Dent 1998;80(1):32-35. DOI: 10.1016/s0022-3913(98) 70088-5.

23. Marafie $\mathrm{Y}$, Looney $\mathrm{S}$, Nelson S, et al. Retention strength of impression materials to a tray material using different adhesive methods. J Prosthet Dent 2008;100(6):432-440. DOI: 10.1016/S00223913(08)60260-7.

24. Chai JY, Jameson LM, Moser JB, et al. Adhesive properties of several impression material systems: Part I. J Prosthet Dent 1991;66(2):201-209. DOI: 10.1016/s0022-3913(05)80048-4. 